\title{
Privatheit vs. Öffentlichkeit
}

Neue Regeln für Berichte aus dem Gerichtssaal

Anna K. Bernzen

\section{Von Tennisspielenden und Medienschaffenden}

Als Tennislegende Boris Becker sich von seiner ersten Ehefrau Barbara Becker scheiden ließ, konnte die interessierte Öffentlichkeit den Scheidungsprozess nicht nur in den einschlägigen Illustrierten verfolgen. Auch Fernsehkameras waren damals im Gerichtssaal im US-amerikanischen Florida präsent. Bis heute ist auf YouTube zum Beispiel ein Video abrufbar, in dem Becker dem Familienrichter mitteilt, wie viele Sicherheitsleute seine Familie rund um die Uhr bewachen. ${ }^{1}$

Hätte der Tennisspieler sich hierzulande scheiden lassen, wären diese privaten Umstände nicht aufgezeichnet worden. In deutschen Gerichtssälen müssen sich Medienschaffende, die Aufnahmen herstellen wollen, schließlich an strengere Regeln halten als in den USA. Diese Regeln, die teilweise bereits in den 1960er Jahren erlassen wurden, passen jedoch nicht mehr in die moderne Medienwelt. Nachdem eingangs der aktuelle Rechtsrahmen für die Arbeit der Medienschaffenden im Gericht beschrieben wurde, werden in diesem Beitrag deshalb neue Regeln für die Berichte aus dem Gerichtssaal vorgeschlagen.

Dabei werden nicht nur Bild/Ton-Aufnahmen wie jene von Beckers Scheidungsprozess betrachtet, die im Fernsehen ausgestrahlt, bei YouTube oder als Instagram-Story veröffentlicht werden können. Es werden vielmehr alle Medienformen berücksichtigt, die eine Live-Berichterstattung aus dem Gerichtssaal ermöglichen. Dies sind daneben Ton-Aufnahmen, die etwa im Radio oder für einen Podcast genutzt werden können, BildAufnahmen, also Fotografien für Zeitungen, Snapchat und Co., und Textberichte in Echtzeit, die bspw. auf Twitter oder über einen Nachrichtenticker publiziert werden.

1 Vgl. YouTube: AP Archive (21.07.2015). 


\section{Aktuelle Regeln für Berichte aus dem Gerichtssaal}

\section{1 Öffentlichkeit mündlicher Verhandlungen}

Die Grundvoraussetzung für die Berichterstattung aus dem Gerichtssaal ist die Öffentlichkeit der Verhandlung, über die berichtet wird. ${ }^{2}$ Sie ist in $\$ 169$ Abs. 1 S. 1 GVG gesetzlich festgeschrieben. Danach können Medienschaffende - ebenso wie ,gewöhnliche' Bürgerinnen - im Grundsatz jeder mündlichen Verhandlung vor einem erkennenden Gericht zusehen und zuhören. ${ }^{3}$ Das Geschehen vor Gericht dürfen sie außerdem für ihre Berichte dokumentieren, etwa mittels Notizen oder Zeichnungen. ${ }^{4}$ Daraus folgt im Umkehrschluss, dass Medienberichte aus einer nichtöffentlichen Verhandlung von vornherein nicht möglich sind. ${ }^{5}$ Eine Grenze wird der Arbeit der Medienschaffenden daher gezogen, wo die Öffentlichkeit aus einer Gerichtsverhandlung ausgeschlossen ist.

Zum Schutz der Privatheit der am Verfahren beteiligten Personen ist ein solcher Öffentlichkeitsausschluss für manche Verhandlungen im Gesetz vorgesehen. In Familiensachen etwa, in denen regelmäßig besonders private Lebensbereiche der Beteiligten thematisiert werden, wird die Öffentlichkeit nach $\mathbb{\$} 170$ Abs. 1 S. 1 GVG von Gesetzes wegen mit dieser Zielrichtung ausgeschlossen. ${ }^{6}$

In anderen Verhandlungen kann das Gericht die Öffentlichkeit jedenfalls im Einzelfall ausschließen. Dies kommt nach $\$ 171$ a GVG etwa in der Hauptverhandlung vor einem Strafgericht in Betracht, die sich mit der Unterbringung der angeklagten Person in einem psychiatrischen Krankenhaus oder einer Entziehungsanstalt befasst. Dadurch soll vermieden werden, dass intime Aspekte wie die psychische Gesundheit der betroffenen Person an die Öffentlichkeit gelangen. ${ }^{7}$

2 Entsprechend Burkhardt/Peifer (2018). In: Wenzel, Wort- und Bildberichterstattung, Kap. 10 Rn. 181.

3 Vgl. nur BVerfG, Urteil vom 24.01.2001 - 1 BvR 2623/95, 622/99, BVerfGE 103, 44 (61); von Coelln, AfP 2014, 193; Schwarz (2015). In: Burkiczak et al., BVerfGG, $\$ 17$ a Rn. 6.

4 Vgl. nur BGH, Urteil vom 15.01.1963 - 5 StR 528/62, BGHSt 18, 179 (181); Burkhardt/Peifer (2018). In: Wenzel, Wort- und Bildberichterstattung, Kap. 10 Rn. 183.

5 Von der theoretischen Möglichkeit, Medienschaffenden nach $₫ 175$ Abs. 2 GVG für ihre Berichterstattung den Zutritt zu nichtöffentlichen Verhandlungen zu gewähren, wird in der Praxis kaum Gebrauch gemacht.

6 Vgl. BTDrucks 16/6308, S. 320.

7 Vgl. Mayer (2018). In: Kissel/Mayer, GVG, $\$ 171$ a Rn. 1. 
Diese Öffentlichkeit kann außerdem nach $\$ 171$ b Abs. 1 GVG ausgeschlossen werden, soweit Umstände aus dem persönlichen Lebensbereich bestimmter beteiligter Personen wie etwa einer Zeugin zur Sprache kommen, deren öffentliche Erörterung schutzwürdige Interessen verletzen würde. Dies setzt allerdings voraus, dass das Interesse an der öffentlichen Erörterung nicht überwiegt. Erfasst sind alle Umstände, „nach denen üblicherweise im Sozialleben nicht gefragt zu werden pflegt und die in der Regel nicht spontan und unbefangen mitgeteilt werden" 8 . Ein Beispiel ist der Gesundheitszustand der betroffenen Person. ${ }^{9}$ Diese privaten Aspekte werden infolge des Öffentlichkeitsausschlusses der Kenntnis der Zuschauenden entzogen. Die Öffentlichkeit ist dabei sogar zwingend auszuschließen, wenn die betroffene Person den Ausschluss beantragt ( $\$ 171$ b Abs. 3 GVG).

Des Weiteren kann die Öffentlichkeit nach $\$ 172$ Nr. 3 GVG ausgeschlossen werden, wenn in der Verhandlung ein privates Geheimnis erörtert wird, dessen unbefugte Offenbarung durch die Zeugin oder die Sachverständige mit Strafe bedroht ist. Dies ist vor allem der Fall, wenn diese Aussagepersonen sich zu privaten Umständen äußern müssen, die sie im Rahmen ihres Berufes erfahren haben, bei dessen Ausübung sie einer strafbewehrten Schweigepflicht unterliegen. Betroffen ist etwa ärztliches oder therapeutisches Personal. ${ }^{10}$

Doch selbst wenn keiner der dargestellten Ausschlussgründe vorliegt und eine Gerichtsverhandlung deshalb öffentlich ist, bedeutet das nicht, dass alle Medienschaffenden uneingeschränkt hieraus berichten können. Während zum Beispiel die Vertreterin einer Tageszeitung mithilfe ihrer Notizen einen anschaulichen Bericht über einen Prozess verfassen kann, reicht die bloße Teilnahme an der Gerichtsverhandlung etwa für Radiound Fernsehreporterinnen nicht aus. Sie müssen ihre Berichte schließlich mit Aufnahmen illustrieren. Die Grenzen, die ihnen bei deren Anfertigung gezogen werden, werden im Folgenden aufgezeigt.

\subsection{Bild/Ton-Aufnabmen und Ton-Aufnahmen}

Bild/Ton-Aufnahmen und Ton-Aufnahmen im Gerichtssaal richten sich nach denselben Regeln. Welche dies konkret sind, hängt davon ab, zu wel-

8 Rieß/Hilger, NStZ 1987, 145 (150).

9 Vgl. Mayer (2018). In: Kissel/Mayer, GVG, $\mathbb{1} 171$ b Rn. 3.

10 Vgl. Mayer (2018). In: Kissel/Mayer, GVG, $\$ 172$ Rn. 45 f. 
chem Zeitpunkt die Aufnahmen angefertigt werden: in der mündlichen Verhandlung oder in deren Umfeld, also vor oder nach der Verhandlung bzw. in deren Pausen.

\subsubsection{Aufnahmen in der mündlichen Verhandlung}

Bild/Ton- und Ton-Aufnahmen sind in der mündlichen Verhandlung nach $₫ 169$ Abs. 1 S. 2 GVG im Grundsatz verboten. Das betrifft alle Aufnahmen am Ort der mündlichen Verhandlung - unabhängig davon, wo diese abgehalten wird. ${ }^{11}$ Bei der Einnahme eines Augenscheins am Tatort greift das Aufnahmeverbot zum Beispiel ebenso wie während der Zeugenvernehmung im Gerichtssaal. Es ist auch irrelevant, ob Aufnahmen sogleich per Livestream übertragen werden oder erst einmal für die spätere Berichterstattung gespeichert werden. ${ }^{12}$ Nicht nur professionelle Medienschaffende müssen sich dabei an das Verbot halten, auch ,Normalbürgerinnen' dürfen die Verhandlung nicht aufnehmen und etwa auf Instagram Live übertragen. ${ }^{13}$ Verboten sind die Aufnahmen aber nur, wenn sie zum Zweck ihrer unmittelbaren öffentlichen Vorführung oder Veröffentlichung ihres Inhalts hergestellt werden. Aufnahmen, die Medienschaffende etwa als Gedächtnisstütze für ihre Berichterstattung anfertigen, sind vom gesetzlichen Verbot dagegen nicht erfasst. ${ }^{14}$

Ausnahmen vom strengen Aufnahmeverbot existieren nur an hohen Bundesgerichten. Am Bundesverfassungsgericht sind die Bild/Ton-Aufnahmen und Ton-Aufnahmen nach $₫ 17$ a Abs. 1 S. 2 BVerfGG zu Beginn der Verhandlung, bis die Anwesenheit der Beteiligten festgestellt wurde, und während der öffentlichen Entscheidungsverkündung ohne eine vorherige Erlaubnis zulässig. Die Vorsitzende Richterin kann Aufnahmen oder ihre Übertragung nach $\$ 17$ a Abs. 2 BVerfGG jedoch verbieten oder Auflagen dafür machen. Dies ist zur Wahrung sowohl des ordnungsgemäßen Verfahrensablaufs als auch der schutzwürdigen Interessen der Verfahrensbetei-

11 Vgl. nur BGH, Urteil vom 17.02.1989 - 2 StR 402/88, BGHSt 36, 119; Bernzen (2020: Kap. 1, B) II) 1) c) cc)); Dannecker (2014: S. 238); Mayer (2018). In: Kissel/ Mayer, GVG, $\$ 169$ Rn. 63.

12 Vgl. nur BTDrucks 18/10144, S.11; Bernzen (2020: Kap. 1, B) II) 1) c) aa) (1)); Krieg, K\&R 2009, 673 (675); Mayer (2018). In: Kissel/Mayer, GVG, $\$ 169$ Rn. 63.

13 Vgl. nur Bernzen (2020: Kap. 1, B) II) 1) c) dd)); Hamm, AfP 2014, 202 (203); Kujath (2011: S. 244); Schwarz, AfP 1995, 353 (355).

14 Vgl. nur Bernzen (2020: Kap. 1, B) II) 1) c) aa) (3)); von Coelln (2005: S. 320); Schwarz (2015). In: Burkiczak et al., BVerfGG, $\mathbb{\$} 17$ a Rn. 19. 
ligten oder Dritter möglich. Die Vorsitzende könnte die Aufnahmen der Entscheidungsverkündung bspw. verbieten, wenn das Gericht über eine Verfassungsbeschwerde befindet und hierfür Umstände aus der engeren persönlichen Lebenssphäre der beschwerdeführenden Person erörtert werden müssen.

Eine Ausnahme vom Aufnahmeverbot kann außerdem an den obersten Bundesgerichten gemacht werden, also am Bundesgerichtshof, Bundesarbeitsgericht, Bundesverwaltungsgericht, Bundessozialgericht sowie Bundesfinanzhof. Nach $\mathbb{1 6 9}$ Abs. 3 S. 1 GVG kann das Gericht dort die Bild/ Ton- und Ton-Aufnahmen während der öffentlichen Entscheidungsverkündung zulassen. Dies ist allerdings nur in besonderen Fällen gestattet. Dies sind Fälle, in denen ein großes öffentliches Informationsinteresse an dem betroffenen Verfahren besteht, das die Medien an das Gericht herantragen. ${ }^{15}$ Einmal erlaubte Aufnahmen oder deren Übertragung können nach $\$ 169$ Abs. 3 S. 2 GVG allerdings unter denselben Voraussetzungen wieder beschränkt werden wie die Aufnahmen am Bundesverfassungsgericht.

\subsubsection{Aufnahmen im Umfeld der mündlichen Verbandlung}

Im Umfeld der mündlichen Verhandlung sind Bild/Ton- und Ton-Aufnahmen mangels eines gesetzlichen Verbotes prinzipiell ohne vorherige richterliche Erlaubnis zulässig. ${ }^{16}$ In dieser Phase werden sie in der Praxis in öffentlichkeitswirksamen Verfahren auch regelmäßig angefertigt, da dies aufgrund des strengen Aufnahmeverbots während der Verhandlung selbst aktuell die einzige Möglichkeit für Medienschaffende ist, Aufnahmen für ihre Berichte über einen Gerichtsprozess herzustellen. ${ }^{17}$

Auch außerhalb der mündlichen Verhandlung sind Bild/Ton- und TonAufnahmen aber nicht unbeschränkt möglich. Die Vorsitzende Richterin kann sie vielmehr auf der Grundlage der sitzungspolizeilichen Generalklausel in $\$ 176$ Abs. 1 GVG nach freiem Ermessen beschränken - also entweder untersagen oder Auflagen hierfür machen. ${ }^{18}$

15 Vgl. Bernzen (2020: Kap. 1, B) II) 3) e) bb)).

16 Vgl. nur Bernzen (2020: Kap. 1, B) III) 1)); Bernzen/Bräutigam, K\&R 2017, 555 (556); Burkhardt/Peifer (2018). In: Wenzel, Wort- und Bildberichterstattung, Kap. 10 Rn. 184.

17 Vgl. Bernzen, NJW 2017, 799 (800).

18 Vgl. schon BTDrucks III/2037, S. 44; BTDrucks IV/178, S. 45 f. 
Bei der Ausübung ihres Ermessens bewegt sie sich jedoch in einem engen Rechtsrahmen, den das Bundesverfassungsgericht in zahlreichen Entscheidungen absteckte. ${ }^{19}$ Es war hierfür zuständig, weil die Aufnahmetätigkeit der Medienschaffenden im Gerichtssaal in den sachlichen Schutzbereich der Medienfreiheiten nach Artikel 5 Abs. 1 S. 2 GG fällt. ${ }^{20}$ Die Presseund die Rundfunkfreiheit garantieren die freie Beschaffung und Verbreitung von Informationen auf die von den Medienschaffenden gewählte Art und Weise. ${ }^{21}$ Daraus folgt, dass die Beschränkung der Aufnahmetätigkeit im Gericht einen Eingriff in die Medienfreiheiten darstellt, an dessen Rechtfertigung besondere Anforderungen zu stellen sind. ${ }^{22}$ Die Vorsitzende Richterin muss bei ihrer Ermessenausübung insbesondere dem Verhältnismäßigkeitsgrundsatz in seiner speziellen Ausprägung der Wechselwirkungslehre entsprechen. Demnach muss ein Gesetz, das die Medienfreiheiten beschränkt, seinerseits im Lichte der Bedeutung der Medienfreiheiten ausgelegt und dadurch in seiner diese Freiheiten beschränkenden Wirkung wiederum eingeschränkt werden. ${ }^{23}$

Das Bundesverfassungsgericht leitete daraus in seinen Entscheidungen als zentrale Vorgabe ab, dass die Vorsitzende Richterin kein pauschales Aufnahmeverbot erlassen darf. ${ }^{24}$ Ein solches Verbot ist aus seiner Sicht insbesondere nicht erforderlich, da diverse mildere Maßnahmen denkbar

19 Wegweisend war hierbei die Entscheidung des Bundesverfassungsgerichts mit Beschluss vom 14.07.1994 - 1 BvR 1595/92, 1606/92, BVerfGE 91, 125. Zuletzt entschied das Bundesverfassungsgericht zu diesem Komplex mit Beschluss vom 09.09.2017 - 1 BvR 2022/16, NJW 2017, 798 sowie mit Beschluss vom 17.08.2017 -1 BvR 1741/17, NJW 2017, 3288.

20 Vgl. ausführlich dazu Bernzen (2020: Kap. 2, C) I) 2) b)). Anderer Ansicht speziell für Bild/Ton-Aufnahmen und Ton-Aufnahmen in der mündlichen Verhandlung jedoch BVerfG, Urteil vom 24.01.2001 - 1 BvR 2623/95, 622/99, BVerfGE 103, 44 (59).

21 Ständige Rechtsprechung, vgl. nur BVerfG, Beschluss vom 06.10.1959 - 1 BvL 118/53, BVerfGE 10, 118 (121); Beschluss vom 14.07.1994 - 1 BvR 1595/92, 1606/92, BVerfGE 91, 125 (135); Urteil vom 27.02.2007 - 1 BvR 538/06, 2045/06, BVerfGE 117, 244 (259).

22 Vgl. Bernzen (2020: Kap. 2, C) I) 4)).

23 Ständige Rechtsprechung, vgl. nur BVerfG, Urteil vom 15.01.1958 - I BvR 400/51, BVerfGE 7, 198 (208f.); Beschluss vom 04.11.2009 - 1 BvR 2150/08, BVerfGE 124, 300 (331 f.); Urteil vom 22.02.2011 - 1 BvR 699/06, BVerfGE 128, 226 (265 f.).

24 Entsprechend BVerfG, Beschluss vom 14.07.1994 - 1 BvR 1595/92, 1606/92, BVerfGE 91, 125 (137-139); Beschluss vom 15.04.2002 - 1 BvR 680/02, NJW 2002, 2021 (2022); Beschluss vom 03.04.2009 - 1 BvR 654/09, NJW 2009, 2117 (2119). 
sind, die gleich wirksam sind. So können mögliche Störungen des äußeren Geschehensablaufs zum Beispiel ebenso effektiv wie durch ein Aufnahmeverbot dadurch vermieden werden, dass eine Pool-Lösung angeordnet wird. Bei dieser in der Praxis oft gewählten Lösung darf ein einziges Kamerateam Aufnahmen im Gerichtssaal anfertigen, die es anschließend an alle interessierten Rundfunksender weitergibt. So kann vermieden werden, dass ein störender ,Medienrummel' entsteht. ${ }^{25}$

Auch mildere Maßnahmen als ein Aufnahmeverbot wie eine solche Auflage darf die Vorsitzende Richterin nach der Rechtsprechung des Bundesverfassungsgerichts allerdings nicht pauschal anordnen. ${ }^{26}$ Sie muss vielmehr im Einzelfall überprüfen, ob diese Maßnahmen angemessen sind, indem sie alle von den Aufnahmen betroffenen Rechte und schutzwürdigen Interessen gegeneinander abwägt. Auf der einen Seite muss sie dabei vor allem das Informationsinteresse der Öffentlichkeit und das hiermit korrespondierende Berichterstattungsinteresse der Medienschaffenden berücksichtigen, die durch die Medienfreiheiten geschützt werden. ${ }^{27}$ Auf der anderen Seite können vor allem das allgemeine Persönlichkeitsrecht der Anwesenden nach Art. 2 Abs. 1 i.V.m. Art. 1 Abs. 1 GG und das rechtsstaatliche Gebot der Funktionstüchtigkeit der Rechtspflege in die Abwägung einzubeziehen sein..$^{28}$

\subsection{Bild-Aufnabmen}

Bild-Aufnahmen sind in Ermangelung eines gesetzlichen Verbotes sowohl in der mündlichen Verhandlung als auch in deren Umfeld im Grundsatz ohne vorherige Erlaubnis zulässig. ${ }^{29}$ In der Praxis werden sie allerdings zu-

25 Vgl. BVerfG, Beschluss vom 14.07.1994 - 1 BvR 1595/92, 1606/92, BVerfGE 91, 125 (137 f.); Beschluss vom 19.12.2007 - 1 BvR 620/07, BVerfGE 119, 309 (325327).

26 Entsprechend BVerfG, Beschluss vom 10.04.2003 - 1 BvR 697/03, NJW 2003, 2523.

27 Vgl. nur BVerfG, Beschluss vom 21.07.2000 - 1 BvQ 17/00, NJW 2000, 2890 (2891); Beschluss vom 27.11.2008 - 1 BvQ 46/08, NJW 2009, 350 (351); Beschluss vom 30.03.2012 - 1 BvR 711/12, NJW 2012, 2178 (2179).

28 Vgl. nur BVerfG, Beschluss vom 14.07.1994 - 1 BvR 1595/92, 1606/92, BVerfGE 91, 125 (136f.); Beschluss vom 19.12.2007 - 1 BvR 620/07, BVerfGE 119, 309 (324f.); Beschluss vom 03.04.2009 - 1 BvR 654/09, NJW 2009, 2117 (2119).

29 Vgl. nur Bernzen (2020: Kap. 1, C) I)); Bernzen/Bräutigam, K\&R 2017, 555 (556); Burkhardt/Peifer (2018). In: Wenzel, Wort- und Bildberichterstattung, Kap. 10 Rn. 184. 
meist im selben Umfang verboten wie Bild/Ton- und Ton-Aufnahmen. Jedenfalls in der mündlichen Verhandlung sind sie demnach kaum einmal zulässig und auch im Umfeld der Verhandlung werden sie häufig entgegen der Gesetzeslage von einer vorherigen Zustimmung abhängig gemacht. ${ }^{30}$

Ebenso wie Bild/Ton- und Ton-Aufnahmen im Umfeld der mündlichen Verhandlung kann die Vorsitzende Richterin auch Bild-Aufnahmen auf der Basis der sitzungspolizeilichen Generalklausel in $\$ 176$ Abs. 1 GVG beschränken. ${ }^{31}$ Allerdings stellt auch diese Beschränkung einen Eingriff in die Medienfreiheiten der Medienschaffenden dar, sodass an ihre Rechtfertigung wiederum besondere Anforderungen zu stellen sind. ${ }^{32}$ Bei der Ausübung ihres Ermessens bewegt die Vorsitzende Richterin sich deshalb erneut in einem vom Bundesverfassungsgericht in diversen Entscheidungen abgesteckten, engen Rahmen. Dieser Rahmen entspricht im Wesentlichen dem, der für Bild/Ton- und Ton-Aufnahmen im Umfeld der mündlichen Verhandlung nachgezeichnet wurde. Die Beschränkung muss mithin insbesondere in jedem Einzelfall verhältnismäßig sein. ${ }^{33}$

\subsection{Textberichte in Echtzeit}

Textberichte in Echtzeit sind ebenso wie Bild-Aufnahmen mangels eines gesetzlichen Verbotes sowohl in der mündlichen Verhandlung als auch in deren Umfeld grundsätzlich ohne Erlaubnis zulässig. ${ }^{34}$ Ebenfalls in Parallele zu Bild-Aufnahmen ist jedoch zu konstatieren, dass Textberichte in Echtzeit in der Praxis jedenfalls während der mündlichen Verhandlung regelmäßig verboten werden. Das Bundesverfassungsgericht etwa sieht in seinen Akkreditierungsbedingungen standardmäßig vor, dass das Twittern und sonstige Versenden von Nachrichten im Sitzungssaal untersagt ist.

30 Vgl. zur divergierenden Handhabung der Gerichte Lehr, NStZ 2001, 63.

31 Vgl. schon BTDrucks III/2037, S. 44; BTDrucks IV/178, S. 45.

32 Vgl. ausführlich dazu erneut Bernzen (2020: Kap. 2, C) I) 2) b), 4)). Dies entspricht - anders als für die Bild/Ton- und Ton-Aufnahmen - für alle Phasen der Berichterstattung aus dem Gerichtssaal auch der ständigen Rechtsprechung des Bundesverfassungsgerichts, vgl. nur BVerfG, Beschluss vom 11.05.1994 - 1 BvR 733/94, NJW 1996, 310; Beschluss vom 31.7.2014 - 1 BvR 1858/14, NJW 2014, 3013 (3014); Beschluss vom 09.09.2016 - 1 BvR 2022/16, NJW 2017, 798.

33 Vgl. für eine ausführliche Darstellung der Rechtsprechung Bernzen (2020: Kap. 1, C) III)).

34 Vgl. nur Bernzen (2020: Kap. 1, D) II) 5)); Krieg, K\&R 2009, 673 (677 f.); Mayer (2018). In: Kissel/Mayer, GVG, $\$ 169$ Rn. 67. 
Medienschaffende dürfen ihre Computer zur Durchsetzung dieses Verbotes nur im Offline-Betrieb und nur auf der Presseempore nutzen. ${ }^{35}$

Die Grundlage für derartige Beschränkungen der Textberichterstattung in Echtzeit liefert der Vorsitzenden Richterin erneut die sitzungspolizeiliche Generalklausel in $\$ 176$ Abs. 1 GVG. ${ }^{36}$ Dabei wird es in ihr freies Ermessen gestellt, auch Textberichte zu verbieten oder Auflagen hierfür vorzusehen. Anders als zu den verschiedenen Aufnahmeformen existiert zu dieser Art der Berichterstattung allerdings noch keine Rechtsprechung des Bundesverfassungsgerichts, die der Vorsitzenden dabei verfassungsrechtliche Grenzen aufzeigt. ${ }^{37}$

\section{Neue Regeln für Berichte aus dem Gerichtssaal}

\subsection{Weiterhin: Öffentlichkeit mündlicher Verhandlungen}

Die Grundvoraussetzung für die Berichterstattung aus dem Gerichtssaal sollte auch zukünftig sein, dass die mündlichen Verhandlungen, über die berichtet wird, öffentlich sind. Insbesondere wenn einer der eingangs dargestellten Ausschlussgründe vorliegt, sollten weiterhin keine Aufnahmen oder Textberichte in Echtzeit im Gerichtssaal möglich sein. Weder sollte künftig deshalb ein Scheidungsprozess aufgezeichnet noch die Verhandlung über die Unterbringung einer drogensüchtigen Person per Livestream verfolgt werden können. Einzig die Regeln, die speziell die Live-Berichterstattung aus dem Gerichtssaal erfassen, sollten einer Reform unterzogen werden.

\subsection{Nur vorläufige Regelung der Berichterstattung aus dem Gerichtssaal}

Die neuen Regeln für Berichte aus dem Gerichtssaal sollten jedoch nur vorläufig sein. Jede Beschränkung dieser Berichterstattung stellt schließlich

35 Vgl. etwa die Akkreditierungsbedingungen für die Urteilsverkündung zum „Streikrecht für Beamte“, BVerfG (08.05.2018).

36 Vgl. Hamm, AfP 2014, 202 (204); Heckmann, AnwZert ITR 15/2012 Anm. 1.

37 Es existieren zwar Entscheidungen zur Nutzung von Laptops und Smartphones im Gerichtssaal (vgl. BVerfG, Beschluss vom 03.12.2008 - 1 BvQ 47/08, NJW 2009, 352; Beschluss vom 31.07.2014 - 1 BvR 1858/14, NJW 2014, 3013). In ihnen ging es aber um klassische Textberichterstattung, die mit diesen Hilfsmitteln zwar erleichtert worden wäre, jedoch auch ohne sie möglich ist. 
- wie gezeigt - einen Eingriff in die Medienfreiheiten dar und muss daher den Vorgaben des Verhältnismäßigkeitsgrundsatzes in seiner besonderen Ausprägung der Wechselwirkungslehre entsprechen. Zuvorderst muss die Beschränkung daher einen legitimen Zweck verfolgen. ${ }^{38}$ Dies kann prinzipiell der Schutz all jener Rechte und schutzwürdiger Interessen sein, die durch Aufnahmen und Textberichte in Echtzeit gefährdet würden. ${ }^{39}$ Beispielhaft sei auf die angesprochenen Risiken für das allgemeine Persönlichkeitsrecht der Anwesenden und die Funktionsfähigkeit der Rechtspflege verwiesen.

Der Schutz dieser Positionen ist jedoch nur dann ein legitimer Zweck für den Eingriff in die Medienfreiheiten, wenn sie durch die Berichterstattung aus dem Gerichtssaal überhaupt gefährdet werden. Dem Gesetzgeber kommt bei der Festlegung des legitimen Zweckes eines Grundrechtseingriffs zwar ein großer Spielraum zu, er kann allerdings auch nicht irgendeinen Zweck wählen, der mit dem Eingriff in keinerlei Zusammenhang steht. ${ }^{40}$ Es ist daher problematisch, dass die von den Gerichtssaalberichten ausgehenden Gefahren bisher nicht empirisch erforscht wurden. ${ }^{41}$

Es existieren zwar einige Positionen, von denen aus rechtlichen Gründen bereits heute sicher ist, dass sie durch Aufnahmen und Textberichte in Echtzeit beeinträchtigt werden können. Dies betrifft etwa die engere persönliche Lebenssphäre, die eine Ausprägung des allgemeinen Persönlichkeitsrechts ist. ${ }^{42}$ Wird zum Beispiel in einem Arzthaftungsprozess der Gesundheitszustand der klagenden Person erörtert, könnten Fernsehaufnahmen hiervon ihre persönlichen Umstände aufgrund der erheblichen Reichweite der Fernsehberichte einem größeren Personenkreis bekannt machen, als es bspw. klassische Zeitungsberichte über das Verfahren könnten. ${ }^{43}$

Für andere Positionen, die der Zulassung der Gerichtssaalberichte in Rechtsprechung und Literatur entgegengehalten werden, ist aus tatsächli-

38 Vgl. zu den einzelnen Anforderungen an die Verhältnismäßigkeit nur BVerfG, Beschluss vom 15.01.1970 - 1 BvR 13/68, BVerfGE 27, 344 (352); Beschluss vom 26.02.2008 - 2 BvR 392/07, BVerfGE 120, 224 (239-241); Urteil vom 27.02.2008 1 BvR 370/07, 595/07, BVerfGE 120, 274 (318 f.).

39 Vgl. Bernzen (2020: Kap. 5, B) III) 2) a)).

40 Vgl. Kluckert, JuS 2015, 116 (121).

41 Von Coelln, AfP 2014, 193 (201) spricht treffend von einer ,gefühlte[n] Selbstverständlichkeit“, mit der ohne wissenschaftliche Erforschung zahlreiche negative Auswirkungen der Gerichtssaalberichterstattung angenommen werden.

42 Vgl. nur BVerfG, Urteil vom 15.12.1999 - 1 BvR 653/96, BVerfGE 101, 361 (382); Beschluss vom 26.02.2008 - 1 BvR 1626/07, BVerfGE 120, 180 (199); Urteil vom 27.02.2008 - 1 BvR 370/07, 595/07, BVerfGE 120, 274 (311).

Entsprechend Bernzen (2020: Kap. 3, B) III) 1) a)). 
chen Gründen dagegen nicht sicher, ob sie durch diese Berichte tatsächlich beeinträchtigt werden. So wird zum Beispiel vermutet, dass insbesondere Aufnahmen die richterliche Entscheidungsfindung nachteilig beeinflussen könnten. Das Gericht könnte, so wird befürchtet, eine psychische Befangenheit verspüren und daher womöglich nicht mehr frei entscheiden. ${ }^{44} \mathrm{Al}$ lerdings existieren zwar medien- und sozialwissenschaftliche Studien, die auf einen Einfluss der klassischen, nachträglichen Berichterstattung über Gerichtsverfahren auf die Unabhängigkeit der beteiligten Richterinnen hindeuten. ${ }^{45}$ Inwiefern der Einfluss der Berichte durch Aufnahmen und Textberichte in Echtzeit gesteigert werden würde, ist dagegen bislang nicht erforscht.

Es ist demnach zwar sicher, dass die Berichterstattung aus dem Gerichtssaal mit Gefahren für einige Rechte und schutzwürdige Interessen einhergeht. Welche dies konkret sind, ist nach aktuellem Forschungsstand dagegen teilweise noch nicht sicher. Diese unklare Sachlage kann einerseits nicht dazu führen, dass zum Schutz der Rechte und Interessen, für die Gefahren (noch) nicht nachgewiesen sind, überhaupt nicht gehandelt werden darf. Würden Richterinnen zum Beispiel durch die Aufnahmen tatsächlich bei ihrer Entscheidungsfindung beeinflusst, würden infolge von deren $\mathrm{Zu}$ lassung womöglich Fehlurteile gefällt. Andererseits streitet der fehlende Nachweis der Risiken für diverse Rechte und Interessen gegen eine endgültige Beschränkung der Medienfreiheiten zum Schutz dieser Positionen.

Auflösen lässt sich dieser Konflikt, indem vorläufig alle denkbaren Gefahren bei einer Zulassung der Aufnahmen und Textberichte in Echtzeit im größten möglichen Umfang unterstellt werden und, darauf basierend, ein enger Rahmen für die Berichterstattung aus dem Gerichtssaal abgesteckt wird. Nach einiger Zeit sollte dieser Rahmen jedoch evaluiert und ggf. in dem Umfang gelockert werden, in dem sich die vermuteten Risiken nicht realisierten. ${ }^{46}$

\subsection{Differenzierende Regelung der Berichterstattung aus dem Gerichtssaal}

Die neuen Regeln sollten zudem zwischen den unterschiedlichen Situationen im Gerichtssaal differenzieren. Auf der einen Seite sollte zwischen den

44 Vgl. nur Dahs, NJW 1961, 1755 (1756); Gerhardt (1968: S. 84); Wickern (2010). In: Löwe/Rosenberg, StPO, Vor $\$ 169$ GVG Rn. 18, $\$ 169$ GVG Rn. 41.

45 Vgl. insbesondere die Studien von Kepplinger et al. (2018); Kepplinger/Zerback, Publizistik 2009, 216 (229-231); Sternberg et al., PVS 56 (2015), 570 (591-594).

46 Vgl. ausführlich zu alledem Bernzen (2020: Kap. 5, B) III) 2) b)). 
Formen der Berichterstattung unterschieden werden, wobei Aufnahmen anders geregelt werden sollten als Textberichte in Echtzeit. Auf der anderen Seite sollte zwischen den Verfahrensarten und den Instanzen differenziert werden.

\subsubsection{Aufnabmen in den Tatsacheninstanzen der Strafverfahren}

Aufnahmen sollten dabei im Grundsatz verboten sein. Das neue Aufnahmeverbot sollte sogar noch weitreichender ausfallen als das derzeitige: In sachlicher Hinsicht sollte es nicht nur Bild/Ton- und Ton-Aufnahmen erfassen, sondern auch reine Bild-Aufnahmen. Von ihnen gehen schließlich vielfach vergleichbare Gefahren für die Rechte und Interessen aus, die eine Beschränkung der Gerichtssaalberichte schützen soll, sodass eine großzügigere Zulassung der Bild-Aufnahmen nicht angemessen ist. Werden zum Beispiel Fotografien persönlicher Gegenstände angefertigt, die bei Gericht in Augenschein genommen wurden, können durch die Veröffentlichung dieser Aufnahmen ebenso private Informationen verbreitet werden wie durch die Publikation entsprechender Bild/Ton-Aufnahmen.

In zeitlicher Hinsicht sollte das Verbot außerdem nicht nur die Verhandlung, sondern auch deren Umfeld erfassen. Auch die Gefahren in diesen beiden Phasen sind schließlich vergleichbar. Für die Frage, ob das Recht der Verfahrensbeteiligten am eigenen Bild durch ungewollte Aufnahmen beeinträchtigt wird, ist es zum Beispiel irrelevant, ob der Prozess bereits begonnen hat oder ob die Beteiligten noch auf das Gericht warten. Auch ist denkbar, dass die Gerichtssaalberichte im Umfeld der mündlichen Verhandlung Auswirkungen auf diese haben. So könnten die Richterinnen auf dem Weg in den Gerichtssaal bspw. von Kamerateams belagert und dadurch so gehemmt werden, dass sie noch in der Verhandlung einen Einfluss auf ihre Entscheidungsfindung verspüren. ${ }^{47}$

Das neue Aufnahmeverbot sollte aber insofern enger sein als das heutige Verbot, als es nur in den Tatsacheninstanzen der Strafverfahren absolut gelten sollte. ${ }^{48}$ Ein Aufnahmeverbot stellt schließlich einerseits die schwerste denkbare Beeinträchtigung der Medienfreiheiten dar, da den Medienschaffenden damit die Informationsbeschaffung und -verbreitung

47 Entsprechend BVerfG, Beschluss vom 19.12.2007 - 1 BvR 620/07, BVerfGE 119, 309 (325).

48 Vgl. ausführlich zu den Tatsacheninstanzen der Strafverfahren Bernzen (2020: Kap. 5, B) III) 5) d) bb)). 
mittels Aufnahmen unmöglich gemacht wird. ${ }^{49}$ Andererseits beeinträchtigt es auch andere für die Zulassung von Aufnahmen streitende Positionen schwer. Dies betrifft insbesondere den Öffentlichkeitsgrundsatz, der auf dem Rechtsstaats- und dem Demokratieprinzip gründet. ${ }^{50}$ Er dient der Kontrolle der Judikative, ${ }^{51}$ er soll der Allgemeinheit helfen, Vertrauen in die Gerichte zu bilden ${ }^{52}$ und sie über ihre Arbeit zu informieren ${ }^{53}$. Diese Funktionen des Öffentlichkeitsgrundsatzes werden durch Aufnahmen besser erfüllt als durch klassische Gerichtsberichte, die etwa in der Zeitung veröffentlicht werden. Das folgt insbesondere aus der größeren Reichweite der Fernsehberichte, die mehr Bürgerinnen das Geschehen bei Gericht zur Kenntnis bringt. ${ }^{54}$ Werden Aufnahmen verboten, können die Zwecke des Öffentlichkeitsgrundsatzes demnach nicht im größten möglichen Umfang verwirklicht werden. ${ }^{55}$

Den schweren Beeinträchtigungen vor allem der Medienfreiheiten und des Öffentlichkeitsgrundsatzes als abstrakt gewichtigen Positionen müssen entsprechend große Gemeinwohlgewinne mit Blick auf diejenigen Positionen entgegenstehen, die durch die Aufnahmen gefährdet werden. Dabei muss berücksichtigt werden, dass Medienschaffende auch bei einem Verbot aller Aufnahmen in der öffentlichen Verhandlung präsent sein und im Anschluss darüber berichten können. Das führt einerseits dazu, dass ein Aufnahmeverbot die Positionen, die für Aufnahmen streiten, weniger schwer beeinträchtigt. Eine Berichterstattung über Gerichtsverfahren wird auch durch ein Aufnahmeverbot schließlich nicht völlig vereitelt. ${ }^{56}$

Andererseits bedeutet es, dass sich für einige der Positionen, die gegen die Aufnahmen sprechen, mit einem Aufnahmeverbot keine großen Gewinne erzielen lassen. Diese Positionen werden schließlich bereits durch die klassische Berichterstattung aus dem Gericht gefährdet, die aufgrund

49 Vgl. Bernzen (2020: Kap. 5, B) III) 5) c) bb) (2) (b), (c)).

50 Vgl. nur BVerfG, Urteil vom 24.01.2001 - 1 BvR 2623/95, BVerfGE 103, 44 (63 f.); Beschluss vom 19.12.2007 - 1 BvR 620/07, BVerfGE 119, 309 (319f.); BVerwG, Urteil vom 01.10.2014 - 6 C 35/13, NJW 2015, 807 (809).

51 Vgl. nur BVerfG, Urteil vom 24.01.2001 - 1 BvR 2623/95, BVerfGE 103, 44 (63 f.).

52 Vgl. nur BVerfG, Beschluss vom 14.03.2012 - 2 BvR 2405/11, NJW 2012, 1863 (1865); RG, Urteil vom 14.02.1936 - 1 D 63/36, RGSt 70, 109 (112); BGH, Urteil vom 17.02.1989 - 2 StR 402/88, BGHSt 36, 119 (122).

53 Vgl. nur BVerfG, Urteil vom 24.01.2001 - 1 BvR 2623/95, BVerfGE 103, 44 (64 f.); Beschluss vom 19.12.2007 - 1 BvR 620/07, BVerfGE 119, 309 (319f.); BGH, Urteil vom 14.06.1994 - 1 StR 40/94, BGHSt 40, 191 (194).

54 Vgl. Bernzen (2020: Kap. 2, B) III) 2) d), 3) c), 4) d)).

55 Vgl. Bernzen (2020: Kap. 5, B) III) 5) c) bb) (3)).

56 Vgl. Bernzen (2020: Kap. 5, B) III) 5) c) bb) (1)). 
der Öffentlichkeit mündlicher Verhandlungen möglich ist. Diese Gefahren müssen aber hingenommen werden, hat sich die Legislative doch für die grundsätzliche Öffentlichkeit der Gerichtsverhandlungen entschieden. Zugunsten einer Beschränkung der Aufnahmen dürfen deshalb nur die Risiken berücksichtigt werden, die über die Nachteile der klassischen Gerichtsberichterstattung hinausgehen. ${ }^{57}$

Mit einem Aufnahmeverbot in den Tatsacheninstanzen der Strafverfahren lassen sich auch unter dieser Prämisse große Gewinne erzielen. Einerseits existieren diverse Positionen, die nur oder jedenfalls vorrangig in den Tatsacheninstanzen gefährdet werden können. Umstände, die der engeren persönlichen Lebenssphäre zuzuordnen sind, werden zum Beispiel vor allem in diesen Instanzen erörtert, weil in ihnen der Sachverhalt ermittelt wird, über den das Gericht zu entscheiden hat. In den Rechtsinstanzen werden dagegen vorwiegend Rechtsfragen geklärt, sodass private Umstände dort allenfalls am Rande zur Sprache kommen. ${ }^{58}$ Andererseits sind in den Strafverfahren Positionen zu berücksichtigen, die in anderen Verfahrensarten von vornherein nicht beeinträchtigt werden können. Dies betrifft etwa das Recht auf Resozialisierung, das als Ausfluss des allgemeinen Persönlichkeitsrechts und des Sozialstaatsprinzips jeder Person, die wegen einer Straftat verurteilt wird, nach Verbüßung der Strafe zusteht. ${ }^{59}$

\subsubsection{Aufnabmen in den Rechtsinstanzen der Strafverfahren und den Tatsacheninstanzen der anderen Verfahrensarten}

In den Rechtsinstanzen der Strafverfahren, aber auch in den Tatsacheninstanzen der anderen Verfahrensarten - also bspw. in zivil- oder arbeitsgerichtlichen Prozessen - sollten Aufnahmen zwar im Grundsatz ebenfalls verboten sein. Die Vorsitzende Richterin sollte im Einzelfall aber eine Ausnahme von dem Aufnahmeverbot machen können. ${ }^{60}$

57 Vgl. Bernzen (2020: Kap. 5, B) III) 5) c) cc) (1)).

58 Vgl. Bernzen (2020: Kap. 3, B) III) 1) b) bb)).

59 Vgl. Bernzen (2020: Kap. 3, B) III) 5)). Vgl. zum Recht auf Resozialisierung nur BVerfG, Urteil vom 05.06.1973 - 1 BvR 536/72, BVerfGE 35, 202 (236); Beschluss vom 18.06.1997 - 2 BvR 483/95, 2501/95, 2990/95, BVerfGE 96, 100 (115); Urteil vom 01.07.1998 - 2 BvR 441, 493/90, 618/92, 212/93, 2 BvL 17/94, BVerfGE 98, 169 (200).

60 Vgl. ausführlich zu den Rechtsinstanzen der Strafverfahren und den Tatsacheninstanzen der anderen Verfahrensarten Bernzen (2020: Kap. 5, B) III) 5) d) cc), dd)). 
Die Notwendigkeit seiner Lockerung folgt für das Strafverfahren daraus, dass Aufnahmen - wie soeben gezeigt - in den Rechtsinstanzen bestimmte Rechte und schutzwürdige Interessen von vornherein nicht oder jedenfalls nicht so schwer wie in den Tatsacheninstanzen beeinträchtigen können. Mit Blick auf die übrigen Verfahrensarten ist zu berücksichtigen, dass die Aufnahmen die strafrechtsspezifischen Positionen, die gegen ihre Zulassung streiten können, von vornherein nicht gefährden können. Ein absolutes Aufnahmeverbot ist daher weder in den Rechtsinstanzen der Strafverfahren noch in den Tatsacheninstanzen der anderen Verfahrensarten angemessen. Dem Schutz der auch unter diesen Umständen gefährdeten Positionen kann vielmehr schon dadurch Rechnung getragen werden, dass die Vorsitzende Richterin die Zulassung der Aufnahmen im Einzelfall verweigern oder Auflagen hierfür machen kann. Werden in einem arbeitsgerichtlichen Prozess zum Beispiel die familiären Umstände der klagenden Person erörtert, die einer Kündigung entgegenstehen, kann die Vorsitzende es zum Schutz der engeren persönlichen Lebenssphäre dieser Person ablehnen, Aufnahmen hiervon zu gestatten.

\subsubsection{Aufnabmen in den Rechtsinstanzen der anderen Verfahrensarten und am Bundesverfassungsgericht}

In den Rechtsinstanzen der Verfahren außerhalb des Strafverfahrens und in den Verfahren am Bundesverfassungsgericht sollten Aufnahmen dagegen im Grundsatz zulässig sein. ${ }^{61}$ In diesen Verfahren werden die Rechte und schutzwürdigen Interessen, die gegen die Zulassung der Aufnahmen sprechen, schließlich regelmäßig in einem noch einmal geringeren Umfang gefährdet als in den bisher dargestellten Verfahren. Weder werden die Positionen gefährdet, die vor allem in den Tatsacheninstanzen zum Tragen kommen, noch bestehen strafverfahrensspezifische Risiken. Ein angemessener Ausgleich zwischen allen betroffenen Rechten und schutzwürdigen Interessen kann daher bereits dadurch erzielt werden, dass die prinzipiell zulässigen Aufnahmen durch die Vorsitzende Richterin im Einzelfall unterbunden oder mit Auflagen versehen werden können, sollten bestimmte Positionen im Einzelfall doch einmal gefährdet sein. Wird in einem Prozess am Bundessozialgericht zum Beispiel die Behinderung der klagenden

61 Vgl. ausführlich zu den Rechtsinstanzen außerhalb des Strafverfahrens und zu den Verfahren am Bundesverfassungsgericht Bernzen (2020: Kap. 5, B) III) 5) d) ee)). 
Person erörtert, können Aufnahmen für diesen Abschnitt der Verhandlung untersagt werden.

\subsubsection{Textberichte in Echtzeit}

Zuletzt sollten die Textberichte in Echtzeit sowohl während der mündlichen Verhandlung als auch in deren Umfeld prinzipiell gestattet sein. ${ }^{62}$ Dies stellt zwar keine Neuerung gegenüber der geltenden Rechtslage dar. Eine gesetzliche Klarstellung ist jedoch geboten, da in der Praxis - wie gezeigt - jedenfalls für die Dauer der Verhandlung häufig ein pauschales Verbot der Textberichterstattung in Echtzeit erfolgt. ${ }^{63}$

Für diese im Vergleich mit den Aufnahmen großzügigere Regelung spricht, dass ein Verbot der Textberichte in Echtzeit die Medienfreiheiten noch einmal schwerer beeinträchtigt als ein Aufnahmeverbot. Ein Fernsehbericht über einen Gerichtsprozess etwa kann auch ohne Aufnahmen aus dem Gerichtssaal angefertigt werden. In der Praxis erzählt eine Korrespondentin hierfür oft das Geschehen vor der Kamera nach. Sind Textberichte in Echtzeit im Gerichtssaal verboten, sind sie dagegen schlechthin unmöglich. Möglich sind nur nachträgliche, zusammenfassende Textberichte, etwa in der Zeitung. Sie sind aber etwas anderes als die zeitgleiche und detaillierte Wiedergabe des Geschehens, die etwa auf Twitter oder über einen Live-Blog möglich ist. ${ }^{64}$

Dem stehen nur geringe Gewinne für den Schutz derjenigen Rechte und schutzwürdigen Interessen gegenüber, die Textberichte in Echtzeit gefährden können. Ein Live-Blog in Textform bedeutet etwa von vornherein keine Gefahr für das Recht der Anwesenden am eigenen Bild. ${ }^{65}$ Auch der äußere Verfahrensablauf wird nicht gestört, wenn Medienschaffende mithilfe ihres Smartphones bloggen. ${ }^{66}$ Die möglichen Gewinne wiegen daher nicht so schwer, dass ein auch nur grundsätzliches Verbot der Textberichte in Echtzeit angemessen wäre. Bestehen im Einzelfall doch einmal Gefahren für bestimmte Positionen, kann die Vorsitzende Richterin die Berichte untersagen oder unter Auflagen stellen. So kann der Einsatz von Twitter und Co. zum Beispiel für die Phase eines finanzgerichtlichen Verfahrens

62 Vgl. ausführlich zu den Textberichten in Echtzeit Bernzen (2020: Kap. 5, B) III) 5) d) ff)).

63 Vgl. Bernzen (2020: Kap. 5, D) V) 1)).

64 Vgl. Bernzen (2020: Kap. 5, B) III) 5) c) bb) (2) (c)).

65 Vgl. Bernzen (2020: Kap. 3, B) III) 2) b)).

66 Vgl. Bernzen (2020: Kap. 3, G)). 
verboten werden, in der die Einkommensverhältnisse einer Privatperson zur Sprache kommen.

\section{Fazit}

Zusammenfassend sollten die rechtlichen Rahmenbedingungen für Gerichtssaalberichte, die seit den 1960er Jahren in weiten Teilen unverändert gelten, reformiert werden. Die Reformvorschrift sollte dabei einerseits nach einem Jahr evaluiert und womöglich gelockert werden. Andererseits sollte sie derart differenzieren, dass Textberichte in Echtzeit im Grundsatz erlaubt und Aufnahmen im Grundsatz verboten sind. Die Vorsitzende sollte das Aufnahmeverbot jedoch in den Rechtsinstanzen der Strafverfahren und in den Tatsacheninstanzen der übrigen Verfahren im Einzelfall aufheben können. In den Rechtsinstanzen der übrigen Verfahren und am Bundesverfassungsgericht schließlich sollen Aufnahmen sogar im Grundsatz erlaubt sein.

\section{Literaturverzeichnis}

Bernzen, Anna K. (2017): „Sitzungspolizeiliche Anordnung zur Bildberichterstattung im Strafverfahren“. In: Neue Juristische Wochenschrift (NJW) 70 (11), S. 799-800.

Bernzen, Anna K. (2020): Gerichtssaalberichterstattung. Ein zeitgemäßer Rahmen für die Arbeit der Medienvertreter in deutschen Gerichten. Tübingen: Mohr Siebeck.

Bernzen, Anna K./Bräutigam, Frank (2017): „Chance statt Risiko: das Gesetz zur Erweiterung der Medienöffentlichkeit in Gerichtsverfahren“. In: Kommunikation \& Recht (K\&R) 20 (9), S. 555-559.

Bundesverfassungsgericht (2018): „Urteilsverkündung in Sachen ,Streikrecht für Beamte' am Dienstag, 12. Juni 2018, 10.00 Uhr. Pressemitteilung Nr. 35/2018 vom 8. Mai 2018“ (08.05.2018). URL: https://www.bundesverfassungsgericht.de/ SharedDocs/Pressemitteilungen/DE/2018/bvg18-035.html [Abruf am: 19.09.2019].

Burkiczak, Christian et al. (Hrsg.) (2015): Bundesverfassungsgerichtsgesetz, Heidelberg: C. F. Müller.

von Coelln, Christian (2005): Zur Medienöffentlichkeit der Dritten Gewalt. Rechtliche Aspekte des Zugangs der Medien zur Rechtsprechung im Verfassungsstaat des Grundgesetzes. Tübingen: Mohr Siebeck. 
von Coelln, Christian (2014): „Justiz und Medien. Rechtliche Anforderungen an das Verhältnis zwischen der Justiz und den Medien, insbesondere an die Berichterstattung über Gerichtsverfahren“. In: AfP - Zeitschrift für Medien- und Kommunikationsrecht (AfP) 40 (3), S. 193-202.

Dahs, Hans (1959): „Der Anwalt im Strafprozeß“. In: Anwaltsblatt (AnwBl) 9 (8/9), S. 171-189.

Dannecker, Gerhard (2014): „Die Öffentlichkeit von Gesetzen“. In: Schoberth, Ingrid (Hrsg.): Urteilen lernen II. Ästhetische, politische und eschatologische Perspektiven moralischer Urteilsbildung im interdisziplinären Diskurs. Göttingen: V\&R Unipress, S. 225-245.

Gerhardt, Rudolf (1968): Zur Frage der Verfassungsmäßigkeit des Verbots von Rundfunk- und Fernsehaufnahmen im Gerichtssaal (\$169 Satz 2 GVG). Universität Frankfurt am Main: Dissertation.

Hamm, Rainer (2014): „Justiz und Medien. Rechtliche Anforderungen an das Verhältnis zwischen der Justiz und den Medien, insbesondere an die Berichterstattung über Strafverfahren“. In: AfP - Zeitschrift für Medien- und Kommunikationsrecht (AfP) 40 (3), S. 202-210.

Heckmann, Dirk (2012): „Editorial“. In: AnwaltZertifikatOnline. IT-Recht (AnwZert ITR) Nr. 15 Anm. 1.

Kepplinger, Hans Mathias et al. (2018): Strafprozesse unter dem Einfluss von Online- und Offline-Medien aus Sicht von Richtern und Staatsanwälten. URL: www.kepplinger.de/files/Ergebnisse_Umfrage_V2_0.pdf [Abruf am 19.09.2019].

Kepplinger, Hans Mathias/Zerback, Thomas (2009): „Der Einfluss der Medien auf Richter und Staatsanwälte. Art, Ausmaß und Entstehung reziproker Effekte“. In: Publizistik 54, S. 216-239.

Kissel, Otto Rudolf (Begr.)/Mayer, Herbert (Fortf.) (2018): Gerichtsverfassungsgesetz. Kommentar. München: C.H. Beck.

Kluckert, Sebastian (2015): „Die Gewichtung von öffentlichen Interessen im Rahmen der Verhältnismäßigkeitsprüfung“. In: Juristische Schulung (JuS) 55 (2), S. 116-121.

Krieg, Henning (2009): „Twittern im Gerichtssaal - The revolution will not be televised“. In: Kommunikation \& Recht (K\&R) 12 (11), S. 673-678.

Kujath, Johanna (2011): Der Laienjournalismus im Internet als Teil der Medienöffentlichkeit im Strafverfahren. Neue Herausforderungen durch die Entwicklung des Web 2.0. Berlin: Duncker \& Humblot.

Lehr, Gernot (2001): „Bildberichterstattung der Medien über Strafverfahren“. In: Neue Zeitschrift für Strafrecht (NStZ) 21 (2), S. 63-67.

Löwe, Ewald (Begr.) et al. (2010): Löwe/Rosenberg. Die Strafprozessordnung und das Gerichtsverfassungsgesetz. Großkommentar, Bd. 10 (GVG; EGGVG). Berlin: De Gruyter.

Rieß, Peter/Hilger, Hans (1987): „Das neue Strafverfahrensrecht. Opferschutzgesetz und Strafverfahrensänderungsgesetz 1987“. In: Neue Zeitschrift für Strafrecht (NStZ) 7 (4), S. 145-157. 
Schwarz, Mathias (1995): „Fernsehöffentlichkeit im Gerichtsverfahren. Zur Frage der Verfassungsgemäßheit des $\mathbb{\$} 169$ Satz 2 Gerichtsverfassungsgesetz (GVG)“. In: AfP - Zeitschrift für Medien- und Kommunikationsrecht (AfP) 21 (1), S. 353-357.

Sternberg, Sebastian et al. (2015): „Zum Einfluss der öffentlichen Meinung auf Entscheidungen des Bundesverfassungsgerichts. Eine Analyse von abstrakten Normenkontrollen sowie Bund-Länder-Streitigkeiten 1974-2010“. In: Politische Vierteljahresschrift (PVS) 56 (2), S. 570-598.

YouTube: AP Archive: „USA: BORIS BECKER DIVORCE HEARING“ (21.07.2015). URL: www.youtube.com/watch?v=n8E4N__FUyk [Abruf am: 19.09.2019].

Wenzel, Egbert (Begr.) et al. (2018): Das Recht der Wort- und Bildberichterstattung. Handbuch des Äußerungsrechts. Köln: Otto Schmidt. 
